\title{
Landmark-Guided Surface Matching and Volumetric Warping for Improved Prostate Biopsy Targeting and Guidance
}

\author{
Steven Haker, Simon K. Warfield, and Clare M.C. Tempany \\ Surgical Planning Laboratory \\ Harvard Medical School and Brigham and Women's Hospital \\ 75 Francis St., Boston, MA 02115 USA \\ haker@bwh.harvard.edu
}

\begin{abstract}
We present a composite method for landmark-guided surface matching and volumetric non-rigid registration, with an application to prostate biopsy. The two-step method, based primarily on finite element and thin-plate spline techniques, consists of a boundary matching process, followed by a volumetric warping step. In practice, the boundary matching method allows for registration of anatomical surfaces, such as prostate gland capsules, in a way that is bijective, i.e. one-to-one and onto. The novelty of this approach is that it allows for the exact matching of pre-specified corresponding landmark points on the two surfaces to be matched. The second step, volumetric warping, is presented as an extension of our previous work in prostate registration, having been improved to address the problem of non-bijectivity (the "flipping" of tetrahedra) which can result from the linear-elastic modelling of the deformation. We discuss the use of our method for the registration of pre-operative magnetic resonance (MR) imaging for improved targeting and visualization during MR-guided prostate biopsy. Although presented within the context of prostate MR image registration, our composite surface matching and volumetric registration method has general applicability to other organs and imaging modalities such as $\mathrm{CT}$ and ultrasound.
\end{abstract}

\section{Introduction}

For the male population of the United States, prostate cancer (PC) is the most common non-cutaneous cancer, affecting one in six in his lifetime, and is the second most common cause of cancer death. Prostate cancer takes the life of nearly 40, 000 men annually in the U.S., and the American Cancer Society predicted that 220900 new cases would occur in the in 2003 [1]. In light of the generally aging populations of industrialized nations, prostate cancer will continue to be a major medical and socioeconomic problem.

Prostate cancer is commonly diagnosed by transrectal ultrasound-guided needle biopsy (TRUS), prompted by either an elevated prostate-specific serum antigen (PSA) level or a palpable nodule. TRUS does not target specific lesions, but rather uses a sextant approach, attempting to sample six representative locations 
in the gland. Studies have shown that in more than $20 \%$ of cancers, at least two biopsy sessions were required to diagnose the tumor, and it is not yet clear how many samples (per session) are necessary to reach a diagnosis. Although increasing the number of random TRUS biopsy samples yields a marginal improvement in detection rate 16918 , only a very small fraction of the gland is sampled with even a dozen needle placements. Thus, it is unlikely that simply increasing the number of samples taken will solve the problem. Clearly, an imaging method capable of demarcating the regions of the gland most likely to harbor cancerous tissue could result in a diminished false negative rate of needle biopsy.

Standard MR imaging can clearly define the prostate and its sub-structures, as well as the rectum, neurovascular bundles and urethra. Endo-rectal coil MRI at 1.5 Tesla $(1.5 \mathrm{~T})$, combined with a multi-coil array, is the method in routine clinical use for staging prostate cancer [17]. Further, several new MR imaging methods have recently shown promise in their ability to detect and characterize prostate cancer. These include MRSI (spectroscopy), T2maps, Line Scan Diffusion Imaging, and MR imaging with dynamic intravenous contrast enhancement (gadolinium). Indeed, we have performed a preliminary study, demonstrating the effectiveness of integration of multiple pre-operative MR image sets into a single image, which we call a summary statistical map [6], with validation based on the biopsy sample pathology.

The goal of this research is to increase the information content of images used by the physician during prostate biopsy, through registration of pre-operative MR imaging with intra-operative imaging. In this way, needle placement can be targeted specifically to those locations most likely to contain cancer. Although presented here in the context of MR-guided prostate biopsy, the methods presented are applicable to ultrasound-guided biopsy as well, and to other procedures, such as CT-guided radiation therapies.

\section{Methods}

Here we describe our method of prostate image registration. The prostate, being composed of soft tissue, can undergo significant shape changes between different imaging sessions. We have found, for example, that significant prostate shape changes occur between pre-operative $1.5 \mathrm{~T}$ endorectal coil imaging, in which the patient is supine, and intra-operative $0.5 \mathrm{~T}$ imaging, during which the patient is in the lithotomy position. This shape change is likely the result of changes in patient position and rectal filling necessitated by the procedures. We have performed a quantitative analysis of this deformation, reported in [14]. For this reason, we have pursued the development of non-rigid registration methods. We have performed [3] a preliminary study to develop and evaluate a finite element based non-rigid registration system. The method was an extension of an algorithm developed for brain registration [10. The methods described here further extend, refine and improve upon this work.

The method involves the following steps general steps: 1.) A 3D tetrahedral model of the entire prostate is created from segmented pre-operative $1.5 \mathrm{~T}$ im- 
ages; 2.) the boundary surface of the prostate capsule is extracted from this tetrahedral mesh and is registered to a corresponding capsule surface obtained from segmented intra-operative images; 3.) the surface point matches from step 2 are used as boundary conditions when solving a finite element-based system of evolution equations which models the volumetric deformation within the gland; 4.) the final volumetric deformation field from step 3 step is used to interpolate pre-operative imaging data. We describe these steps in greater detail below.

\subsection{Creation of a Tetrahedral Model of the Pre-operative Prostate}

The starting point of our method is a segmentation of the prostate gland from high-quality pre-operative MR imaging. This segmentation may be obtained by contouring of the gland by hand, or by an automated segmentation method. The surface is extracted as a triangulated surface using the marching cubes algorithm. To create a tetrahedral mesh within this capsular surface, we register to it a highquality meshing of the unit ball $\left\{(x, y, z) \mid x^{2}+y^{2}+z^{2} \leq 1\right\}$. The procedure for this registration is the same as is used to register the pre-operative prostate to the intra-operative prostate, i.e. a surface matching procedure followed by a volumetric warp.

\subsection{Boundary Surface Matching Using Landmark Points}

Our original method for boundary matching [310] used an "active surface model." This method moved one surface toward the other through space, according to a specified attraction force between the surfaces, balanced by an internal energy force used to keep the moving surface smooth and topologically consistent. Difficulties arise from the need to balance these forces, the requirement of a bijective surface matching (a problem in practice), and from the desire for operating-room compatible speed.

We have now improved the speed and robustness of the algorithm by replacing with a direct approach based on our work in the theory of conformal (angle-preserving) mappings [12,2] of surfaces with spherical topology. In this method the surface of the prostate capsule is modeled as a thin elastic sheet which conforms to the altered shape of the capsule as given by the intra-operative imaging. Regardless of the degree of shape deformation, or changes from convexity to concavity, the method yields a one to one mapping of one surface onto another. Further, the method has several inherent advantages over our earlier methods which make it more suitable for use in the operating room. In particular, the core of the algorithm requires only the solution of a pair of sparse linear systems of equations, and thus can be made to run quickly enough to be practical. Indeed, this is the sense in which the method is direct, as the matching from pre-operative surface to inter-operative surface does not require the calculation of intermediate sets of points moving through space. The method also lends itself well to multi-processor parallelization, and standard parallel versions of linear solvers can be used to reduce the solution time significantly. 
In order to find a conformal mapping $u$, we need to solve Laplace's equation,

$$
\nabla^{2} u=0
$$

on the triangulated surface to be registered. Here $\nabla^{2}$ is the Laplace-Beltrami operator, a generalization of the standard second-order Laplacian operator. The trick is to specify a condition, similar to a boundary condition, which results in a $u$ which is conformal. See 122 for details. Although the conformal mapping method produces a matching of surfaces, it is not possible to use conformal mapping alone to match surfaces while, at the same time, requiring that the mapping take a general number of pre-specified landmark points on one surface to the corresponding points on the other. What we have now developed is a method which augments the conformal mapping with a simple thin-plate spline technique [4] to produce a mapping with the desired point-matching property. The use of this method allows for far more control over the final surface match. We typically use as landmark points the 6 points of intersection of the major axes of rotation of the prostate surface with the surface itself. See Fig. 1 .
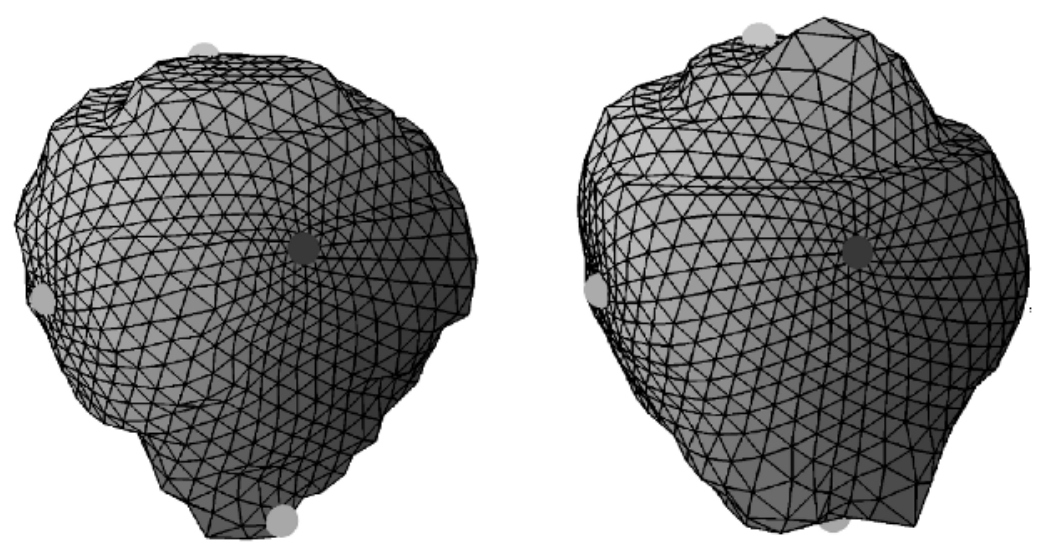

Fig. 1. Two prostate surfaces. The surfaces have been mapped to the sphere and retriangulated in the same way to establish point correspondence. The small spheres represent landmark points which were matched during the registration process.

To see how this is done, let $u_{1}$ and $u_{2}$ be conformal mappings from two surfaces to the unit sphere, and let $w_{1}$ and $w_{2}$ be the corresponding conformal mappings to the plane obtained by composing $u_{1}$ and $u_{2}$ with stereographic projection, a standard map projection used in cartography. Let $s$ be the standard $2 \mathrm{D}$ thin-plate spline mapping that takes a specified set of landmark points in the planar image of $w_{1}$ to the corresponding points in the image of $w_{2}$. Then $z:=w_{2}^{-1} \circ s \circ w_{1}$ is a landmark-matching mapping from one surface to the other. Here $\circ$ represents composition of functions. In practice, the second surface is the usually the sphere itself, with phantom "landmark" points such as the 
poles, $(0,0, \pm 1)$, etc. specified thereon. We can map any prostate surface to the sphere this way, and thus any prostate surface to any other, using compositions of mappings to the sphere and the inverses of these mappings, all the while matching corresponding landmark points. See Fig. 11.

When mapping to the sphere, we use the resulting $z$ mapping as an initial condition for the geometric heat equation

$$
\frac{\partial}{\partial t} z=\nabla^{2} z
$$

while fixing the matched landmark points and re-projecting the evolving surface back to the sphere. This is iterated until near convergence, and helps to produce a smoother, harmonic mapping. Although [11 suggest the use of this type of Jacobi relaxation for finding conformal mappings to the sphere, it is in general too slow to be practical [15], and in fact global long-term solutions to (2) do not in general exist for arbitrary initial conditions and surfaces [19. The reason it is practical for our use is that we have an initial condition that is already close to the final harmonic mapping, due to the use of our conformal mapping technique and perhaps to the intimate relation between thin plate splines and the bi-harmonic equation $\nabla^{4} z=0$.

We note, as an important caveat, that as with standard thin-plate spline methods, we have no guarantee that the final mapping produced will be bijective. We can report however, that in the more than 100 prostate cases that we have processed this way, we have not found the method to fail to produce a bijective mapping.

\subsection{Background on Volumetric Warping}

Given two prostate surfaces, obtained from segmentations of pre-operative and intra-operative images, we may map them to the sphere using the surface warping technique described above, while sending landmark points to pre-specified locations. By interpolating the resulting spherical maps at the vertices of a standard triangulation of the sphere, we can effectively re-triangulate the original prostate surfaces in a consistent manner, thereby providing a point-by-point registration of the surfaces. We use a spherical triangulation which is the boundary of the high-quality tetrahedral meshing of the unit ball, as mentioned in sub-section 2.1. See Fig. 1. We now describe how we use this surface matching to obtain a volumetric registration of the entire interior of the prostate gland.

As mentioned above, in previous work we have performed [3] a preliminary study to develop and evaluate a finite element based non-rigid registration system. The method was an extension of an algorithm developed for brain registration [10]. In this model, the prostate tissue is considered as a linear elastic material. The second-order partial differential equation governing the $3 \mathrm{D}$ deformation field $p$ of the material under this model is

$$
\nabla^{2} p+\frac{1}{1-2 \nu} \nabla \operatorname{div} p=0
$$


subject to our specified boundary conditions. The use of the above equation has, of course, been the subject of investigations by several other research groups, see in particular the pioneering work [7] for an excellent discussion and application to medical image registration. Here Poisson's ratio $\nu$ is constant for a given tissue type, and governs the elastic properties of the deforming material. In 3 we reported that values of $\nu=0.2 \mathrm{kPa}$ for the central gland and $\nu=0.4 \mathrm{kPa}$ for the peripheral zone produced satisfactory results.

The tetrahedral mesh within the prostate surface defines the set of finite elements used to discretize a variational form of the partial differential equation above. The volumetric deformation field is found by solving for the displacement field that minimizes an associated energy. The finite element technique reduces the problem to the solution of a sparse system of linear equations that can be solved for the unknown displacements at each interior vertex.

\subsection{Improving the Volumetric Warping}

We have experienced some problems in practice using the basic method for volumetric warping described above. Specifically, we have found that tetrahedra can "flip" i.e turn inside out in certain cases, and thereby yield a non-bijective registration. This is especially true when some portion of the deforming surface goes from being convex to concave. This may well be the case in practice, as the prostate consists of soft tissue, and the the patient may be scanned using an endo-rectal coil in one imaging session, and with no such coil in another. The reason for this appears to be that the well-known assumption of small deformation required in order for equation (3) to be valid is not being satisfied. Large deformations also cast doubt on the validity of the solution even when no tetrahedra flip.

To address this problem, we have incorporated the following simple technique. Let us suppose we have found the surface mapping between prostate capsules obtained by the conformal mapping and thin plate spline technique described above. Using the surface point correspondence, we align the surfaces with a rigid transformation to minimize the sum-of-squares distance between them. Let $s_{1}=s_{1}(x, y, z)$ be the resulting surface mapping between the prostate capsules after rigid registration. Introduce an artificial time parameter $t$ and create a sequence of $N+1$ surfaces $s(x, y, x, t)=(1-t) \times(x, y, z)+t \times s_{1}(x, y, z)$, for $t=0,1 / N, 2 / N, \ldots, 1$. We can choose $N$ large enough so that the pointwise change in $s$ from one time to another is small with respect to the size of the tetrahedra in the mesh. At each time $t=0,1, \ldots,(N-1) / N$, we compute the volumetric warping using the method described in the previous sub-section with boundary conditions specified by the steps in $s$, arriving in the end with a boundary which is the target capsule surface. We emphasize that it is essential here to re-compute, after each iteration, the matrix associated with the linear system of equations obtained from the finite-element discretization of equation (3). For our application, we have found a value of $N=20$ to be sufficient. 

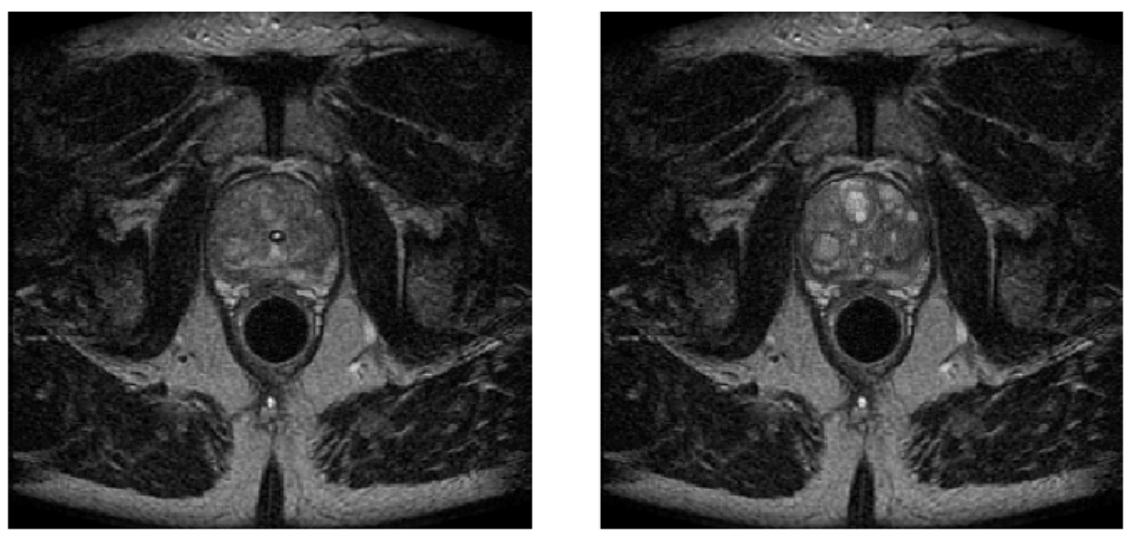

Fig. 2. On the left, an intra-operative $0.5 \mathrm{~T} \mathrm{~T} 2$-weighted axial image of the prostate gland. On the right, within the gland, registered pre-operative $1.5 \mathrm{~T}$ T2-weighted image data. Note the increased conspicuity of the prostate sub-structure; the urethra is not visible due to the absence of a Foley catheter.

In practice, we have found that the above technique eliminates the problem of tetrahedral flipping in all but the most extreme cases. The drawback, of course, is the increased computation time that is necessary.

\subsection{Warping of Pre-operative Images}

After the registration process is complete, we use the resulting mapping to interpolate the pre-operative MR image data into the space of the intra-operative images. Since our method only registers material inside the prostate capsule, we simply take as values outside the gland the data from the intra-operative images. See Fig. 2

\subsection{Rapid Solution of the System of Equations}

In order for this strategy of obtaining a volumetric deformation field to be practical during surgery, we must be able to obtain the solution at a rate compatible with surgical decision making. Fortunately, since the heart of our algorithms involve the solution to linear systems of equations, there are many tools available within the scientific computing community which make this possible. In particular, we have investigated solving the system of linear equations on a set of parallel architectures. See [20 for details. We have recently experimented with both an inexpensive workstation, a Dell Precision 650n with dual 3.0 GHz Intel Xeon CPUs running Linux, and a cluster of such workstations connected by $100 \mathrm{Mbps}$ Fast Ethernet. This has enabled extremely rapid solution of the typical system of equations on inexpensive and widely available hardware, which holds out the possibility of widespread deployment of this technology. With such a set-up, we have found that the solution of (3) can be found in approximately 3 seconds, or $3 \times N$ seconds for the multi-step method of Subsection (2.4). 


\section{Results and Discussion}

We regularly use the algorithm described in the above sections for registration of various MR prostate images, having done so on more than 100 cases to date. In particular, it is now run regularly as part of our MR-guided prostate biopsy procedure [13. The mapping technique allows us to provide the physician with an integrated view of the intra-operative and high quality pre-operative imaging during the biopsy procedure. Fig. 2.

Although presented here for MR, the method has direct applicability to other less discriminating but widely used intra-operative imaging modalities such as CT and ultrasound, since the method does not require intensity information from within the target gland. We plan to test the algorithm's use for these modalities in the near future. Potential uses of the landmark-guided surface registration method include the registration of organs other than the prostate, and applications such as the creation of models of organ variability using principle component analysis. Finally, we mention that in the case where intra-operative images do contain information that might be useful for registration within the gland, we have experimented with using our method as an initial registration step to be improved through a mutual-information maximization process [ 8 ]

Acknowledgements. This work was funded by NIH grants R01 AG 19513-03 and P41 RR13218-06.

\section{References}

1. American Cancer Society. Cancer facts and figures. Atlanta GA, 2003.

2. Angenent S, Haker S, Tannenbaum A, Kikinis R. Laplace-Beltrami operator and brain surface flattening. IEEE Trans. On Medical Imaging, 1999; 18:700-711.

3. Bharatha A, Hirose M, Hata N, Warfield SK, Ferrant M, Zou KH, Suarez-Santana E, Ruiz-Alzola J, D'Amico A, Cormack R, Kikinis R, Jolesz FA, Tempany CM. Evaluation of three-dimensional finite element-based deformable registration of pre- and intra-operative prostate imaging. Med Phys 28: 2551-2560, 2001.

4. Bookstein FL. Size and shape spaces for landmark data in to dimensions (with discussion). Statist. Sci. 1 (1986), 181-242.

5. Brechbuhler C, Gerig G, Kubler O. Parametrization of closed surfaces for 3-D shape description. CVGIP: Image Under 61 (1995) 154-170.

6. Chan I, Wells W III, Mulkern RV, Haker S, Zhang J, Zou KH, Maier SE, Tempany CMC. Detection of prostate cancer by integration of line-scan diffusion, T2mapping and T2-weighted MR imaging; a multi-channel statistical classifier Med Phys, 30 (9) September 2003, pp. 2390-2398.

7. Christensen, G. Deformable shape models for anatomy, Ph.D. thesis, University of Washington, 1994.

8. du Bois d'Aische A, De Craene M, Haker S, Weisenfeld N, Tempany CMC, Macq B, and Warfield SK. Improved nonrigid registration of prostate MRI, MICCAI 2004, submitted.

9. Epstein JI, Walsh PC, Sauvagerot J, et al. Use of repeat sextant and transition zone biopsies for assessing extent of prostate cancer. J Urol 158; 18861997. 
10. Ferrant M, Nabavi A, Macq B, Jolesz FA, Kikinis R, and Warfield SK. 2001. Registration of 3-D intraoperative MR images of the brain using a finite-element biomechanical model. IEEE Trans Med Imaging 20 (12):1384-97.

11. Gu X, Wang Y, Chan T, Thompson P, Yau ST. Genus Zero Surface Conformal Mapping and Its Application to Brain Surface Mapping. IEEE Trans. Med. Image, vol. 23 (7), 2004.

12. Haker S, Angenent S, Tannenbaum A, Halle M, Kikinis R. Conformal surface parameterization for texture mappings. IEEE Trans. On Visualization and Computer Graphics, 2000 April-June.

13. Hata N, Jinzaki M, Kacher D, Cormack R, Gering D, Nabavi A, Silverman SG, D'Amico AV, Kikinis R, Jolesz FA, Tempany CMC. MRI-guided prostate biopsy using surgical navigation software: device validation and feasibility. Radiology, 220:263-268, 2001.

14. Hirose M, Bharatha A, Hata N, Zou K, Warfield S, Cormack R, D'Amico A, Kikinis R, Jolesz F, Tempany C. Quantitative MR imaging assessment of prostate gland deformation before and during MR imaging-guided brachytherapy, Acad. Rad., 9, 8, pp. 906-912. 2002.

15. Press W, Teukolsky S, Vetterling W and Flannery B. Numerical Recipes in C: The Art of Scientific Computing, 2nd Edition, Cambridge University Press, Cambridge U.K., 1992.

16. Rabbani R, Stroumbakis N, Kava BR, Incidence and clinical significance of false negative sextant prostate biopsies. J Urol 1998; 159: 1247.

17. Seltzer SE, Getty DJ, Tempany CMC, Pickett RM, Schnall MD, McNeil BJ, Swets JA. Staging Prostate cancer with MR imaging: A combined radiologist-computer system. Radiology 1997; 202: 219-226.

18. Stroumbakis N, Cookson MS, Reuter V. et al. Clinical significance of repeat sextant biopsies in prostate cancer patients. Urology Suppl 49; 1131997.

19. Struwe M. On the evolution of harmonic mappings of Riemannian surfaces. Comment. Math. Helv. 60 (1985), no. 4, 558-581.

20. Warfield S K, Ferrant M, Gallez X, Nabavi A, Jolesz F, and Kikinis R. Real-Time Biomechanical Simulation of Volumetric Brain Deformation for Image Guided Neurosurgery. In SC 2000: High Performance Networking and Computing Conference; 2000 Nov 4-10; Dallas, USA. 